\title{
Chemoresistive Sensors for Cancer Pre-Screening in Human Tissue
}

\author{
Michele Astolfi ${ }^{1}$, Gabriele Anania ${ }^{1}$, Mascia Benedusi ${ }^{1}$, Sandro Gherardi ${ }^{2}$, Vincenzo Guidi ${ }^{1}$, Nicolò \\ Landini $^{1,2}$, Caterina Palmonari ${ }^{1}$, Giorgio Rispoli ${ }^{1}$, Paola Secchiero ${ }^{1}$, Veronica Tisato ${ }^{1}$, Stefania Gallo ${ }^{1}$, \\ Matteo Valt ${ }^{1}$, Giulia Zonta ${ }^{1,2}$, Cesare Malagù ${ }^{1,2}$ \\ ${ }^{1}$ University of Ferrara, Via Savonarola 9 - 44121 Ferrara, Italy \\ ${ }^{2}$ SCENT S.r.I., Via Quadrifoglio 11 - 44124 Ferrara, Italy \\ ${ }^{3}$ Department of Public Health (AUSL) - UO Igiene Pubblica - Via Fausto Beretta, 7 - 44121 Ferrara, Italy \\ michele.astolfi@unife.it
}

\begin{abstract}
:
One of the greatest goals in medicine is early-stage detection of tumors, to allow physicians and surgeons to apply the therapies available, which are usually successful on small volume cancers only. Our purpose is to identify the presence of a cancer by detecting the metabolites exhaled by cancer cells (VOCs) that are different by the ones exhaled by healthy cells, through a chemoresistive sensor array. In this feasibility study, this fast-responding sensing technique proved to discriminate cancer cells from the healthy one, reliably and reproducibly, making it a good cancer screener with low invasiveness. The measures have been performed on cancer and healthy tissues coming from human colon and rectum.
\end{abstract}

Key words: Chemoresistive, Sensors, Cancer, Biopsy, Metabolites.

\section{Introduction}

Neoplastic tissues exhibit altered metabolic processes with respect to the metabolism of healthy cells, therefore the chemicals (metabolites) expelled during cellular respiration depend upon the cell health status. In this study, a device named SCENT B1 [1] is under development with the aim to discriminate between normal and malignant tissues, by using four chemoresistive sensors (manufactured in the Sensor Laboratory of the University of Ferrara). The electrical signals generated by the sensing unit are amplified, acquired and analyzed by a custom-made hardware and software.

\section{Materials and Methods}

The chemoresistive sensors used in this study are nanostructured semiconductor metal-oxide films (nanograins with average size of 40-50 $\mathrm{nm}$ ). In details, SCENT B1 hosts two sensors composed by a mixture of tin and titanium oxides with the addition of small amounts of gold (ST25), one sensor composed by pure tungsten oxide (WO3), and one sensor composed by pure tin oxide with addition of small amounts of gold (S11).
The voltage output of each sensor, is proportional to its conductance and in turn depends upon the chemicals interacting with its surface [2][5]. Fig. 1 and Fig. 3 show the ratio $\Delta G / G$, where $\Delta G$ is the difference between the sensor conductance with and without the metabolites expelled by cells of a tissue.

Healthy and neoplastic tissues were surgically removed from colon or rectum of informed human subjects and were provided by the Department of Morphology, Surgery and Experimental Medicine of the University of Ferrara. An explanted sample was divided into two pieces of the same weight, one containing the tumor only and one containing healthy cells only, the latter collected as far as possible from the tumor. The tissue samples were placed in Petri dishes containing a standard cell culture medium (DMEM) and stored in an incubator at a temperature of $37-37.5^{\circ}$, to be conserved safe throughout the experiment. The samples were measured in the sequence: i) DMEM only ii) healthy tissue, iii) tumor tissue repeated at least twice to ensure the repeatability of the measures. Healthy and tumor tissues were measured 3 hours (label $A$ in Fig. 1) and 5 hours after the surgery (label B), to monitor any possible time dependence of cell exhalations. 
All four sensors gave larger responses (although with different amplitudes) to tumor tissue with respect to the healthy one. Smaller responses were given by the DMEM only (Fig. 1 and Fig. 3). Table in Fig. 2 underlines the latter concept, expressed in terms of a difference in sensor responses between DMEM samples and healthy or tumor. This response pattern was substantially independent of the time after sample explant (tissues labelled with $A$ or B in Fig. 1 and Fig. 3). These results are consistent with the stronger metabolism of tumor cells with respect to the healthy ones, because the former emits larger amounts of volatile compounds [3]. The diverse responses of each sensor to the same volatile compounds is promising, because more dedicated sensors and a deeper data analysis and statistics could better discriminate the tumor cells from the healthy ones. In principle, this method could be extended to the case of tumors other than in the colon and the rectum.

\section{Conclusions}

This experimental work is still in a preliminary stage, and a larger number of experiments are required to produce a robust statistic. However, the measurements already performed are encouraging, focusing on two different response trends between healthy and tumoraffected tissues. This will be a starting point for an in-depth study for the development of an innovative methodology in the analysis of tumor tissues.

\section{Illustrations}

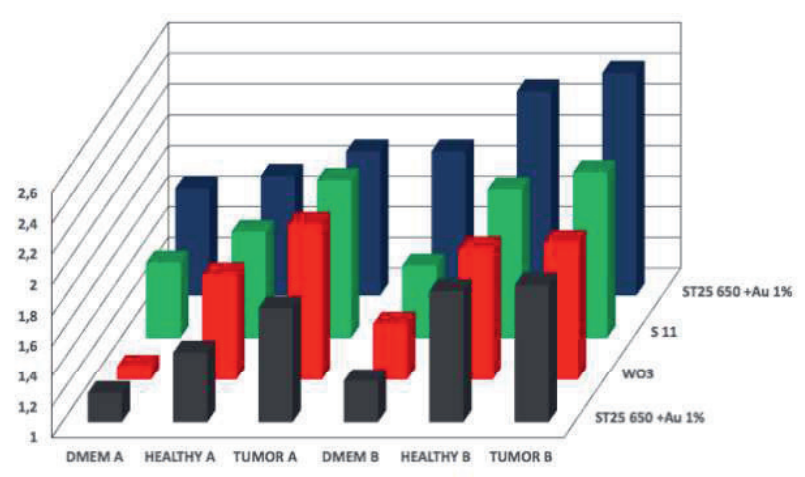

Fig. 1. 3D-histogram of the responses of four different sensors to the cell samples exhalations.

\begin{tabular}{|l|r|r|r|r|r|}
\hline \multicolumn{6}{|c|}{ Difference in Percentage of Responses With Respect to DMEM } \\
\hline SENSORS & HEALTHYA & TUMORA & HEALTHY B & TUMOR B \\
\hline 1) ST25 650+Au 1\% & $22 \%$ & $47 \%$ & $46 \%$ & $49 \%$ \\
\hline 2) WO3 & $55 \%$ & $85 \%$ & $35 \%$ & $38 \%$ \\
\hline 3) S11 & $13 \%$ & $36 \%$ & $34 \%$ & $41 \%$ \\
\hline 4) ST25 650 +Au 1\% & $5 \%$ & $14 \%$ & $20 \%$ & $26 \%$ \\
\hline
\end{tabular}

Fig. 2. Table of the percentage differences among responses upon DMEM and healthy or tumor tissue samples of 3D-histogram in Fig. 1.

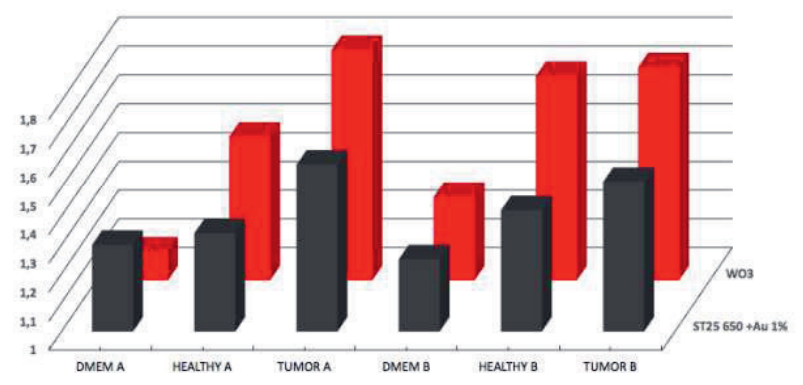

Fig. 3. 3D-histogram focusing upon the responses of two well-responding different sensors (ST25 650 +Au $1 \%$ and WO3) to the cell samples exhalations.

J.K., Keun, H.C., 2009. "Metabolic Profiling of Human Colorectal Cancer Using High-Resolution Magic Angle Spinning Nuclear Magnetic Resonance (HR-MAS NMR) Spectroscopy and Gas Chromatography Mass Spectrometry (GC/MS)", Journal of Proteome Research 8(1), 352-361;

[5] G. Zonta, G. Anania, B. Fabbri, A. Gaiardo, S. Gherardi, A. Giberti, N. Landini, C. Malagù, L. Scagliarini, V. Guidi, Preventive screening of colorectal cancer with a device based on chemoresistive sensors. Sensors and Actuators B, 238, 1098-110, 2016. 